\title{
The Impact of Non-Compliance to a Standardized Risk-Adjusted Protocol on Recurrence, Progression, and Mortality in Non-Muscle Invasive Bladder Cancer
}

This article was published in the following Dove Press journal: Cancer Management and Research

\author{
Faris Abushamma ${ }^{1-3}$ \\ Zain Khayyat' \\ Aya Soroghle' \\ Sa'ed H Zyoud ${ }^{4,5}$ \\ Ahmad Jaradat $\mathbb{D}^{1,2}$ \\ Maha Akkawi ${ }^{1,6}$ \\ Hanood Aburass (D) ${ }^{1,6}$ \\ lyad KK Qaddumi ${ }^{7}$ \\ Razan Odeh ${ }^{8}$ \\ Husam Salameh ${ }^{8}$ \\ Salah Albuheissi ${ }^{3}$
}

'Department of Medicine, College of Medicine and Health Sciences, An-Najah National University, Nablus, 44839, Palestine; ${ }^{2}$ Department of Urology, AnNajah National University Hospital, Nablus, 44839, Palestine; ${ }^{3}$ Bristol Urological Institute, North Bristol NHS Trust, Bristol, UK; ${ }^{4}$ Department of Clinical and Community Pharmacy, College of Medicine and Health Sciences, an-Najah National University, Nablus, 44839, Palestine; ${ }^{5}$ Clinical Research Center, an-Najah National University Hospital, Nablus, 44839, Palestine; ${ }^{6}$ Department of Pathology, An-Najah National University Hospital, Nablus, 44839, Palestine; ${ }^{7}$ Department of Urology, Rafedia General Hospital, Nablus, 44839, Palestine; ${ }^{8}$ Department of Oncology, An-Najah National University Hospital, Nablus, 44839, Palestine

Correspondence: Faris Abushamma Department of Medicine, College of Medicine and Health Sciences, an-Najah National University, Nablus, 44839, Palestine

Email farisabushamma@hotmail.com
Purpose: Non-muscle invasive bladder cancer (NMIBC) is a potentially curable or controllable disease if strict adherence to a surveillance protocol is followed. Management and surveillance of NMIBC begins at the time of diagnosis up to a few years thereafter. There is scanty data in the literature evaluating the impact of non-compliance with the surveillance protocols on progression, recurrence, and mortality rate.

Patients and Methods: An observational, retrospective cohort study recruited data between 2012 and 2017 at two tertiary hospitals. Data were collected consecutively. NMIBC patients who had at least 3 years of follow-up data were included. Patients were divided into different groups based on their compliance with the cystoscopy follow-up protocol as recommended by the European guidelines. We compared the cystoscopy compliant group with the non-compliant group in view of recurrence, progression, and mortality. In addition, missing variable items during surveillance were calculated using a new scoring model to predict adverse outcomes.

Results: Eighty-eight NMIBC patients met our criteria. Recurrence rate (RR), progression rate $(\mathrm{PR})$, metastasis rate (MsR), and mortality rate (MR) are significantly higher in non-compliant group, RR: (92.6\%) $(P<0.001)$, PR: $(54.1 \%)(P<0.001)$, MsR: $(37.7 \%)(P<0.001)$, MR: $(23.5 \%)$ $(P=0.002)$ respectively. In the subgroup analysis, intermediate and high-risk groups have a PR rate of zero in the compliant group, while it is $100 \%(P<0.001)$ and $56.4 \%(P=0.001)$ in the noncompliant group, respectively. Use of a Kaplan Meier (KM) graph shows that compliant patients had a better survival in comparison to non-compliant patients. Scoring there or more is statistically and clinically significantly associated with higher recurrence, progression, and mortality. RR: $(94 \%)(P=0.016)$, PR: $49 \%(P<0.001)$ and MR $(26 \%)(P=0.012)$.

Conclusion: Non-compliance to a standardized surveillance protocol in NMIBC is associated statistically and clinically with adverse outcomes in comparison to a compliant group. This mandates strict adherence to surveillance guidelines particularly in patients with highrisk disease.

Keywords: NMIBC, COVID-19, urological malignancies, haematuria

\section{Introduction}

Bladder cancer is the seventh most common cancer in males and the eleventh most common cancer across both genders. ${ }^{1}$ In Europe, bladder cancer is the 5 th most commonly diagnosed cancer and the 9 th leading cause of mortality. ${ }^{2}$ However, bladder cancer is a broad category disease in view of surveillance and treatment. Non-muscleinvasive bladder cancer (NMIBC) represents the main category, as $75 \%$ of patients with newly diagnosed bladder cancer have non-muscle-invasive pathology. ${ }^{3}$ The goldstandard treatment of NMIBC is based on risk stratification groups in regard to number 
of cystoscopies and intravesical therapy, as recommended by professional organisation guidelines such as the EAU. ${ }^{4}$ Several studies published the natural history of NMIBC mainly in regards to recurrence and progression. This led to the creation of a scoring model by the European Association of Urology (EAU) to predict risk based on the number of tumours, tumour diameter, prior recurrence, concurrent Carcinoma in situ (CIS), and pathological stage and grade. ${ }^{4,5}$

There is strong evidence to support the importance of strict adherence to a surveillance protocol in NMIBC in order to decrease recurrence and progression, and to improve cancer-specific survival. ${ }^{4}$ A number of published articles have investigated the outcome of delayed treatment in muscle-invasive bladder cancer (MIBC) and showed a strong correlation between delaying treatment, and worse outcome and survival. ${ }^{6}$ However, the literature is sparse regarding the effect of non-adherence to a standardized protocol in NMIBC, or the impact of delaying either diagnosis or surveillance cystoscopies. ${ }^{7} \mathrm{~A}$ few published articles investigated the mechanisms responsible for the delays in bladder cancer diagnosis and initial treatment; however, these did not examine the possible adverse outcomes. ${ }^{8}$ The paucity of data regarding the effect of delaying NMIBC management urged us to raise this question. This is particularly well timed as during the current COVID-19 pandemic several papers have been published recommending delaying NMIBC treatment and surveillance, especially in the low-risk category. ${ }^{9,10}$ Therefore, this study aims to identify the impact of nonadherence to standardized risk-adjusted surveillance and intravesical treatment on recurrence rate (RR), progression rate (PR), and mortality rate (MR).

\section{Patients and Methods Study Design}

An observational, retrospective cohort study design was used to evaluate the impact of non- compliance with standardized, risk-adjusted surveillance and treatment in NMIBC. We have used the EAU risk stratification and surveillance protocol as a reference, as this is widely accepted and used in most European centres. ${ }^{11}$

\section{Study Setting}

The data was collected from two tertiary urology centres treating bladder cancer in Northern Palestine, which cover a total population of 500,000 .

\section{Study Population and Sampling}

We reviewed 20,000 histopathological reports between 2012 and 2017. Two hundred bladder biopsies and resection reports were extracted.

\section{Inclusion and Exclusion Criteria Inclusion Criteria}

NMIBC confirmed pathology upon first re-resection during the first 3 months of diagnosis and at least three years of follow-up.

\section{Exclusion Criteria}

Non-urothelial cell carcinoma pathology, less than 12 months of follow-up, muscle invasive bladder cancer (MIBC), lacking mental or physical capacity to communicate with the interviewer, and possible upper tract TCC on contrast imaging.

\section{Data Collection}

NMIBC patients who met our criteria were split into either low, intermediate, or high-risk groups. We also classified them into different groups depending on patient compliance to surveillance cystoscopies:

(A) Group 0 (Compliant):

Low and intermediate risk: Missing zero cystoscopies over the three-year period.

High-risk: Missing one cystoscopy over the three-year period.

(B) Group 1 (Non-compliant 1):

Low and intermediate risk: Missing one cystoscopy over the three-year period.

High risk: Missing two cystoscopies over the three-year period.

(C) Group 2 (Non-compliant 2):

Low and intermediate: Missing two or more cystoscopies over the three year period.

High risk: Missing three or more cystoscopies over the three-year period.

Comparisons between compliant and non-compliant groups were made in order to assess any variance in adverse outcomes (RR over 3 years, PR over 3 years (Progression defined as muscle invasion, prostatic stroma invasion, or development of regional lymphadenopathy on CT scan)). In addition, the metastatic rate (MsR) and MR (Non-cancer-specific) were also evaluated. Compliance to Bacille Calmette-Guérin (BCG) 
instillation treatment was classified as completing 6 cycles of induction and at least the first maintenance cycle.

\section{Statistical Analysis}

All analysis was performed with IBM Statistical Package for Social Sciences program (SPSS) Statistics version 21.0. Categorical variables were presented as absolute frequency (percentage). The Pearson's Chisquare test or Fisher's exact test (as appropriate) were applied to assess the differences in categorical variables. $P$ values $<0.05$ were considered statistically significant. Kaplan-Meier (KM) techniques were used to generate mortality probabilities among adherent versus nonadherent patients in the surveillance protocol for highrisk NMIBC at each consecutive month until 5 years post-diagnosis. A scoring system was created to assess the effect of missing different variables during the surveillance of high-risk patients with $R R, P R$, and MR. The scoring system composed of 4 items extracted from the EAU surveillance protocol for the high-risk group as clarified in Table 1.

\section{Ethical Approval and Consent to Participate}

This study has been approved by the Institutional Review Board of An-Najah National University, and approval from the Palestinian Ministry of Health. The IRB approval can be provided if required. (Archived number 7 Jan 2020). Informed consent was waived by the IRB due to the retrospective chart review and. The study was carried out in compliance with declaration of Helsinki. There is no risk of confidentiality, as the analysis used

Table I Scoring System for High-Risk Group

\begin{tabular}{|l|l|}
\hline Variables & Yes $=\mathbf{0}$ or No $=\mathbf{~ I}$ \\
\hline (3 year follow-up) & \\
Number of missed cystoscopies: & $\begin{array}{l}0: \text { No missing or I } \\
\text { I: } 2 \text { missing } \\
2: 3 \text { missing }\end{array}$ \\
\hline IV-MMC & 0 or I \\
Re-resection at (4-6 weeks) & 0 or I \\
\hline BCG (Induction and at least MI) & 0 or I \\
\hline Total & $<3$ or $\geq 3$ \\
\hline
\end{tabular}

Abbreviations: BCG, Bacille Calmette-Guérin; IV-MMC, intravesical mitomycin. anonymous clinical data and the data cannot be linked to the participating patients.

\section{Results}

\section{Demographic and Clinical Presentation}

Eighty-eight NMIBC patients met our criteria. The mean age of diagnosis was 60 years old $(\mathrm{M}=60, \mathrm{SD}$ : 3.45$)$. The male gender is predominate as $78(88.6 \%)$ were males. The most common presentation was haematuria (visible haematuria (VH) and non-visible haematuria (NVH)), affecting 63 patients $(71.6 \%)$. Half of the patients had the diagnosis made after 2 weeks (M: 8.3, SD: 19.2) (Table 2A).

\section{Staging, Grading and Risk Stratification}

Sixty-six patients (75\%) had a lack of standardized pathological reports but received diagnosis vaguely described as papillary NMIBC. However, for the majority of participants $(66,75 \%)$, their pathology was classed as high grade (G2-3), and this was accurately recorded; $46.6 \%$ had muscle tissue sampled during the first resection, and the rest of the cohort had a confirmatory NMIBC diagnosis during re-resection during the first 3 months post-diagnosis.

The patients were distributed into three main risk group categories according to the EAU risk stratification. ${ }^{11}$ The largest group was the high-risk group which contained 63 patients $(71.6 \%)$, while the low and intermediate group was comprised of 25 patients $(28.4 \%)$ (Table $2 \mathrm{~B})$.

\section{General Compliance with Surveillance Cystoscopy, RR, PR, MsR, and MR}

Generally, most of the cohort are not compliant with the surveillance cystoscopy protocol, with only 20 patients (23\%) classed as compliant. More than $80 \%$ had a recurrence of tumours over the 3 years of follow-up. Progression was noticed in $34(38.6 \%)$ but $8(9 \%)$ had no data available regarding progression. The $\mathrm{MR}$ is around 18\% (Table 2C).

\section{Compliant versus Non-Compliant}

RR, PR, MsR and MR are significantly higher in noncompliant group, RR: (92.6\%) $(P<0.001)$, PR: (54.1\%) $(P<0.001)$, MsR: $(37.7 \%)(P<0.001)$, MR: $(23.5 \%)(P=$ $0.002)$ respectively. Patients older than 60 years old were also significantly less compliant with surveillance cystoscopy protocol $56(82 \%)(P=0.001)$ (Table 3$)$. 
Table 2 Sociodemographic and Clinical Characteristics of Participants $(n=88)$

\begin{tabular}{|c|c|}
\hline Variables & $\begin{array}{l}\text { Number of } \\
\text { Participants (\%) }\end{array}$ \\
\hline \multicolumn{2}{|c|}{ A: Demographic and clinical presentation } \\
\hline \multicolumn{2}{|l|}{ Age } \\
\hline$<60$ & $23(26.1)$ \\
\hline$\geq 60$ & $65(73.9)$ \\
\hline \multicolumn{2}{|l|}{ Gender } \\
\hline Male & $78(88.6)$ \\
\hline Female & $10(11.4)$ \\
\hline \multicolumn{2}{|l|}{ Smoking } \\
\hline Yes & $73(83.0)$ \\
\hline No & $15(17.0)$ \\
\hline \multicolumn{2}{|l|}{ Family history } \\
\hline Yes & $17(19.3)$ \\
\hline No & $7 I(80.7)$ \\
\hline \multicolumn{2}{|l|}{ Co-morbidities (HTN, DM, or IHD) } \\
\hline Yes & $65(73.9)$ \\
\hline No & $23(26.1)$ \\
\hline \multicolumn{2}{|l|}{ Presentation of disease } \\
\hline Haematuria & $63(71.6)$ \\
\hline Scans (Ultrasound, CT scan, etc) & $16(18.2)$ \\
\hline LUTS (mainly dysuria) & $5(5.7)$ \\
\hline Others & $4(4.5)$ \\
\hline \multicolumn{2}{|c|}{ B: Risk stratification, staging and grading } \\
\hline \multicolumn{2}{|l|}{ Stage of tumour } \\
\hline $\mathrm{pTa}$ & $12(13.6)$ \\
\hline PTI & $7(8.0)$ \\
\hline CIS & $3(3.4)$ \\
\hline $\begin{array}{l}\text { General terminology (Superficial } \\
\text { bladder cancer) }\end{array}$ & $66(75.0)$ \\
\hline \multicolumn{2}{|l|}{ Grade of tumour } \\
\hline Low/PUNLMP & $22(25.0)$ \\
\hline High & $66(75.0)$ \\
\hline \multicolumn{2}{|l|}{ Risk group } \\
\hline Low & $14(15.9)$ \\
\hline Intermediate & $\mathrm{II}(\mathrm{I} 2.5)$ \\
\hline High & $63(71.6)$ \\
\hline \multicolumn{2}{|l|}{ Delay of diagnosis } \\
\hline Yes & $44(50.0)$ \\
\hline No & $44(50.0)$ \\
\hline \multicolumn{2}{|l|}{ Muscles found in the first resection } \\
\hline Yes & $4 I(46.6)$ \\
\hline No & $47(53.4)$ \\
\hline
\end{tabular}

(Continued)
Table 2 (Continued).

\begin{tabular}{|l|l|}
\hline Variables & $\begin{array}{l}\text { Number of } \\
\text { Participants (\%) }\end{array}$ \\
\hline $\begin{array}{l}\text { IV-MMC was given at I } \\
\text { Yes }\end{array}$ & $9(10.2)$ \\
No & $79(89.8)$ \\
\hline C: Compliance, RR, PR, MsR and MR Follow over 3 years \\
\hline $\begin{array}{l}\text { Compliance for all groups } \\
\text { Yes }\end{array}$ & $20(22.7)$ \\
No & $68(77.3)$ \\
\hline $\begin{array}{l}\text { Compliance Rate for each risk } \\
\text { Low } \\
\text { Intermediate }\end{array}$ & $6(42.9)$ \\
High & $5(45.5)$ \\
\hline RR & $9(14.3)$ \\
\hline $\begin{array}{l}\text { Yes } \\
\text { No }\end{array}$ & $74(84.1)$ \\
\hline PR & $14(15.9)$ \\
Yes & \\
No & \\
Missing & $34(38.6)$ \\
\hline MetsR & $46(52.3)$ \\
No & $8(9.1)$ \\
\hline Missing & $24(27.3)$ \\
\hline
\end{tabular}

Abbreviations: LUTS, lower urinary tract symptoms; CT, computed tomography scan; BCG, Bacillus Calmette-Guérin; IV-MMC, intravesical mitomycin; DM, diabetes mellitus; HTN, hypertension; IHD, ischemic heart disease; RR, recurrence rate; PR, progression rate; MetsR, metastatic rate; MR, mortality rate; CIS, carcinoma in situ; PUNLMP, papillary urothelial neoplasm of low malignant potential.

\section{Risk Group Subanalysis (Compliance and Relation to RR, PR, and MR)}

The major statistical difference is seen when comparing Group 0 (compliant) to group 2 (non-compliant 2). In particular, non-compliant 2 high-risk group had PR of $56.4 \% \quad(P=0.001)$ and MR of $25 \% \quad(P=0.03)$. Noncompliant 2 intermediate-risk group had RR of $100 \%$ $(P=0.013)$ and $\mathrm{PR}$ of $100 \%(P<0.001)$ (Table 4).

\section{High-Risk Group Compliance to BCG and Cystoscopy}

Non-compliance to both cystoscopy and BCG in the highrisk group is significantly associated with higher PR $42 \%$ 
Table 3 Sociodemographic and Clinical Aspects in Correlation with Compliance

\begin{tabular}{|c|c|c|c|c|}
\hline Variables & Compliant $\mathbf{N}=\mathbf{2 0}(\%)$ & Non-Compliant $\mathbf{N}=68$ & Total & P value ${ }^{a}$ \\
\hline $\begin{array}{l}\text { Gender } \\
\text { Male } \\
\text { Female }\end{array}$ & $\begin{array}{l}19(95 \%) \\
\text { I (5\%) }\end{array}$ & $\begin{array}{l}59 \text { (86.8\%) } \\
9(13.2 \%)\end{array}$ & $\begin{array}{l}78(88.6 \%) \\
10(11.4 \%)\end{array}$ & $0.270^{\mathrm{b}}$ \\
\hline $\begin{array}{l}\text { Age } \\
\qquad 660 \\
>60\end{array}$ & $\begin{array}{l}\text { II (55\%) } \\
9(45 \%)\end{array}$ & $\begin{array}{l}12(17.6 \%) \\
56(82.0 \%)\end{array}$ & $\begin{array}{l}23(26.1 \%) \\
65(73.9 \%)\end{array}$ & $0.00 I^{c}$ \\
\hline $\begin{array}{l}\text { Smoking } \\
\text { Yes } \\
\text { No }\end{array}$ & $\begin{array}{l}18(90 \%) \\
2(20 \%)\end{array}$ & $\begin{array}{l}55(80.9 \%) \\
13(19.1 \%)\end{array}$ & $\begin{array}{l}73(83.0 \%) \\
15(17.0 \%)\end{array}$ & $0.317^{b}$ \\
\hline $\begin{array}{l}\text { Family history } \\
\text { Yes } \\
\text { No }\end{array}$ & $\begin{array}{l}20(100 \%) \\
0(0.0 \%)\end{array}$ & $\begin{array}{l}52(76.5 \%) \\
16(23.5 \%)\end{array}$ & $\begin{array}{l}72(81.8 \%) \\
16(18.2 \%)\end{array}$ & $0.002^{b}$ \\
\hline Co-morbidities & $12(60.0 \%)$ & 53 (77.9\%) & 65 (73.9\%) & $0.108^{c}$ \\
\hline $\begin{array}{l}\text { Delay of diagnosis } \\
\text { (Within } 2 \text { weeks of presentation) }\end{array}$ & $\begin{array}{l}16(80.0 \%) \\
4(20.0 \%)\end{array}$ & $\begin{array}{l}47(69.1 \%) \\
21(30.9 \%)\end{array}$ & $\begin{array}{l}63(71.6 \%) \\
25(28.4 \%)\end{array}$ & $0.002^{c}$ \\
\hline $\begin{array}{l}\text { RR } \\
\text { Yes } \\
\text { No }\end{array}$ & $\begin{array}{l}\text { II (55.0\%) } \\
9(45.0 \%)\end{array}$ & $\begin{array}{l}63(92.6 \%) \\
5(7.4 \%)\end{array}$ & $\begin{array}{l}74(84.1 \%) \\
14(15.9 \%)\end{array}$ & $<0.001^{c}$ \\
\hline $\begin{array}{l}\text { PR } \\
\text { Yes } \\
\text { No }\end{array}$ & $\begin{array}{l}\mathrm{I}(5.3 \%) \\
\mathrm{I} 8(94.7 \%)\end{array}$ & $\begin{array}{l}33(54.1 \%) \\
28(45.9 \%)\end{array}$ & $\begin{array}{l}34(42.5) \\
46(57.5)\end{array}$ & $<0.001^{b}$ \\
\hline $\begin{array}{c}\text { MetsR } \\
\text { Yes } \\
\text { No }\end{array}$ & $\begin{array}{l}\mathrm{I}(5.3 \%) \\
\mathrm{I} 8(94.7 \%)\end{array}$ & $\begin{array}{l}23(37.7 \%) \\
38(62.3 \%)\end{array}$ & $\begin{array}{l}24(30.0 \%) \\
56(70.0 \%)\end{array}$ & $0.003^{b}$ \\
\hline MR & $0(0.0 \%)$ & $16(23.5 \%)$ & $16(18.2 \%)$ & $0.002^{b}$ \\
\hline
\end{tabular}

Notes: a $P$ values in bold are below the significance level of 0.05 . ' $S$ tatistical significance values calculated using Fisher's Exact Test. 'Statistical significance values calculated using the Pearson Chi-Square.

Abbreviations: $R R$, recurrence rate; $P R$, progression rate; $M R$, mortality rate.

$(\mathrm{P}<0.05)$ and higher MR 29\% $(\mathrm{P}<0.05)$ when compared to patients compliant to both (Table 5).

\section{High-Risk Group Cystoscopy Non-Compliance and BCG Therapy}

There is no statistical difference between the noncompliant high-risk group who were having BCG treatment to those not having BCG treatment in regards to $R R$, PR and MR (Table 6).

\section{KM Curve (High-Risk Group)}

Forty-five patients were diagnosed with NMIBC between January 2012 and May 2015. Among these patients, 7 were compliant and 38 were non-compliant. We reviewed the follow-up data for these patients until May 2020. Fifteen deaths were found and all of these were among the noncompliant cohort. To conclude, KM estimator graphing shows that compliant patients were more likely to survive for longer post-diagnosis than non-compliant patients. This finding did not reach statistical significance $(P=0.075)$ because all deceased patients were non-compliant (Figure 1).

\section{Scoring System to Predict Recurrence, Progression, and Mortality in High-Risk Patients with Poor Compliance}

A scoring system has been created to assess the effect of missing different variables during the surveillance of highrisk patients with RR, PR, and MR. The sixty-three 
Table 4 The Correlation Between Compliance in Each Risk Group and RR, PR, and MR

\begin{tabular}{|l|c|c|c|c|c|c|c|c|c|}
\hline \multirow{2}{*}{$\begin{array}{l}\text { Risk } \\
\text { Compliance }\end{array}$} & \multicolumn{3}{|c|}{ Low Risk } & \multicolumn{3}{|c|}{ Intermediate Risk } & \multicolumn{3}{c|}{ High Risk } \\
\cline { 2 - 10 } & RR (\%) & PR (\%) & MR (\%) & RR (\%) & PR (\%) & MR (\%) & RR (\%) & PR (\%) & MR (\%) \\
\hline Compliant & 33.3 & 16.7 & 0.0 & 40.0 & 0.0 & 0.0 & 77.8 & 0.0 & 0.0 \\
Non-compliant & 100.0 & 83.3 & 28.6 & 100.0 & 100.0 & 16.7 & 93.2 & 56.4 & 25.0 \\
P value $^{\mathrm{a}}$ & $\mathbf{0 . 0 0 4}^{\mathrm{b}}$ & $\mathbf{0 . 0 1 6 ^ { \mathrm { b } }}$ & 0.095 & $\mathbf{0 . 0 1 3 ^ { \mathrm { b } }}$ & $<\mathbf{0 . 0 0}^{\mathrm{b}}$ & 0.255 & 0.195 & $\mathbf{0 . 0 0 1}^{\mathrm{b}}$ & $\mathbf{0 . 0 3}^{\mathrm{b}}$ \\
\hline
\end{tabular}

Notes: ${ }^{\text {a }} P$ values in bold are below the significance level of 0.05 . 'S Statistical significance values calculated using Fisher's Exact Test.

Abbreviations: $\mathrm{RR}$, recurrence rate; $\mathrm{PR}$, progression rate; $\mathrm{MR}$, mortality rate.

Table 5 The Correlation Between Compliance to Both Cystoscopy and BCG to RR, PR, MetsR and MR in High-Risk Group

\begin{tabular}{|l|c|c|c|c|c|}
\hline & RR & PR & MetsR & MR & Total \\
\hline Compliant to cystoscopy and BCG & $6(75 \%)$ & $0(0 \%)$ & I (I2.5\%) & $0(0 \%)$ & 8 \\
Non-compliant to either cystoscopy or BCG & $20(95.2 \%)$ & $8(42.1 \%)$ & $8(42.1 \%)$ & $6(28.6 \%)$ & 21 \\
Total & 26 & 8 & 9 & 6 & $0.035^{\mathbf{b}}$ \\
P value $^{\mathrm{a}}$ & $0.133^{\mathrm{b}}$ & $\mathbf{0 . 0 0 8 ^ { \mathrm { b } }}$ & $0.15^{\mathrm{b}}$ & $\mathbf{0 . 1}$ \\
\hline
\end{tabular}

Notes: ${ }^{a} P$ values in bold are below the significance level of 0.05 . ' $S$ tatistical significance values calculated using Fisher's Exact Test.

Abbreviations: BCG, Bacille Calmette-Guérin; RR, recurrence rate; PR, progression rate; MetsR, metastatic rate; MR, mortality rate.

Table 6 The Correlation Between BCG Compliance to RR, PR, MetsR and MR in Non-Compliant Patients in High-Risk Group

\begin{tabular}{|l|c|c|c|c|c|}
\hline & RR & PR & MetsR & MR & Total \\
\hline Compliant to cystoscopy and BCG & $6(75 \%)$ & $0(0 \%)$ & $1(12.5 \%)$ & $0(0 \%)$ & 8 \\
Compliant to BCG & $29(87.9 \%)$ & $14(46.7 \%)$ & $12(40 \%)$ & $7(21.2 \%)$ & 33 \\
Non-compliant to BCG & $20(95.2 \%)$ & $8(42.1 \%)$ & $8(42.1 \%)$ & $6(28.6 \%)$ & 21 \\
Total & 49 & 22 & 20 & 13 & $0.537^{\mathrm{b}}$ \\
P value & $0.363^{\mathrm{a}}$ & $0.754^{\mathrm{b}}$ & $0.884^{\mathrm{b}}$ & \\
\hline
\end{tabular}

Notes: ${ }^{a}$ Statistical significance values calculated using the Pearson Chi-Square. 'Statistical significance values calculated using Fisher's Exact Test.

Abbreviations: BCG, Bacille Calmette-Guérin; RR, recurrence rate; PR, progression rate; MetsR, metastatic rate; MR, mortality rate.

patients from the high-risk group were assessed regarding their compliance with the EAU high-risk surveillance protocol. This includes procedures and treatments, which have been proven to have an impact on recurrence, progression, and mortality. Therefore, we assessed the statistical significance of having a score of three or more in relation to RR, PR, and MR. Scoring three or more is statistically and clinically significantly associated with higher recurrence, progression, and mortality. RR: (94\%) $(P=0.016)$, PR: $49 \%(P<0.001)$ and MR $(26 \%)(P=0.012)$ (Table 7).

\section{Discussion}

This article clearly shows that the PR and RR of NMIBC is significantly higher in the non-compliant group. This is strongly evident in the high-risk subcategory as noncompliance to the cystoscopy surveillance protocol is associated with around $50 \%$ PR and $25 \%$ MR. These results are concomitant to the available evidence as recent data showed that classification as intermediate- or highrisk NMIBC was an independent predictor of progression. ${ }^{12}$ This confirms the importance of following a validated surveillance protocol and not delaying surveillance cystoscopies particularly in the high-risk group.

Several guidelines and recommendations have been used to manage and monitor NMIBC by different international institutions. However, the frequency and duration of follow-up recommendations are based on low levels of evidence, which is illustrated by clear differences in these recommendations per guideline. ${ }^{13}$ Generally, our results showed that poor compliance to cystoscopy surveillance is associated with higher RR, PR and MR regardless of the risk category.

EAU annually updates the guidance on NMIBC surveillance and management, in addition to creating a scoring model to predict progression and recurrence, which are the most important factors in NMIBC prognosis. ${ }^{11}$ However, no previous studies were published 


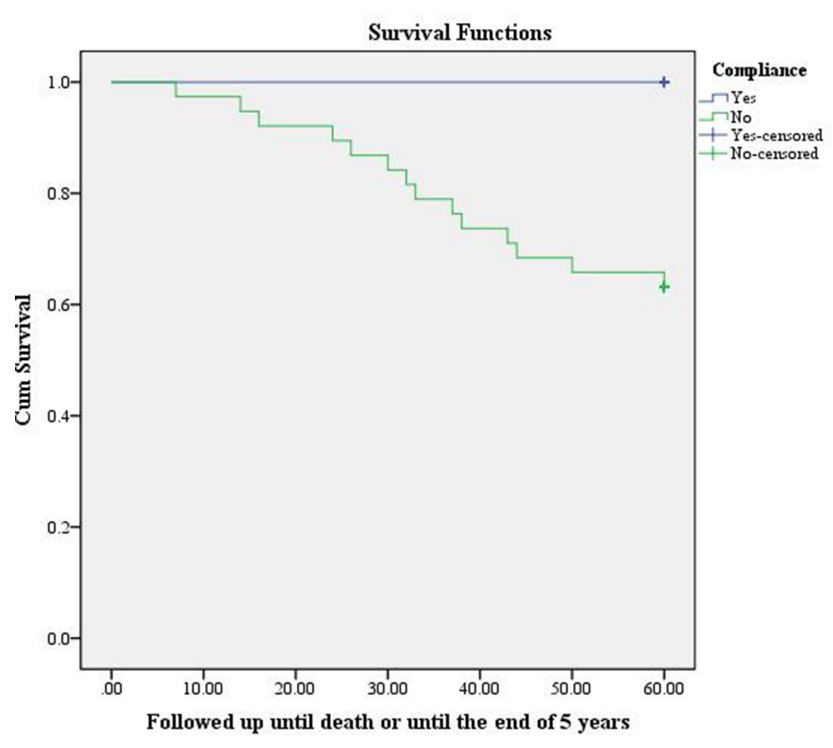

Figure I Comparison of the estimated probability of mortality among compliant versus non-compliant patients in the follow-up of NMIBC based on the KaplanMeier method for 5 years post-diagnosis.

to show the negative impact of missing different variables throughout the NMIBC treatment and surveillance journey. Our postulated scoring model showed that scoring three or more is associated with an adverse outcome. This tool can be used in patient counselling at the diagnosis of NMIBC, so patients could have an insight regarding the possible harm from missing treatment steps during this long and exhausting journey.

A few articles recently raised the question of the overuse of cystoscopy in low-risk NMIBC and its impact on cost and hospital occupancy. On the other hand, underuse in high-risk disease may be associated with serious adverse outcomes. Therefore, a large debate has been raised recently regarding the strict adherence to surveillance cystoscopies taking into account that NMIBC disease is a wide spectrum disease ranging from low risk to high risk, with no available current data regarding the impact of non-compliance to a standardized risk-adjusted

Table 7 Novel Scoring System to Predict Recurrence, Progression and Mortality in the High-Risk Group

\begin{tabular}{|l|c|c|c|}
\hline Outcome & Number of $<\mathbf{3}$ & Number of $\geq \mathbf{3}$ & \multirow{2}{*}{ P value $^{\mathbf{a}}$} \\
\cline { 2 - 3 } & $\mathbf{n}=\mathbf{~ I ~ 2 ( \% ) ~}$ & $\mathbf{n = 5 2 ( \% )}$ & \\
\hline Recurrence & $8(67.0)$ & $48(94.1)$ & $\mathbf{0 . 0 1 6}^{\mathrm{b}}$ \\
Progression & $0(0.0)$ & $25(49)$ & $<0.000 \mathbf{I}^{\mathrm{b}}$ \\
Mortality & $0(0.0)$ & $13(25.5)$ & $\mathbf{0 . 0 1 2}^{\mathrm{b}}$ \\
\hline
\end{tabular}

Notes: ${ }^{a} p$ values in bold are below the significance level of 0.05 . ${ }^{\text {b Statistical }}$ significance values calculated using Fisher's Exact Test. protocol on recurrence, progression and mortality. ${ }^{14,15}$ This evolving concept may harbour hidden harm if not well studied, especially in the high-risk group. This is the group most commonly associated with recurrence and progression according to the EAU scoring prediction model, and is the group most prevalent in our study $(71.5 \%){ }^{11,14}$ Furthermore, there is no available data to look comprehensively into the effect of non-compliance on the bladder cancer diagnosis and management pathway, which also takes into account the time of diagnosis, imaging, cytology, and intravesical therapy. Our data shows that the non-compliant NMIBC group has a higher RR, $\mathrm{PR}$, and MR. This outcome supports the importance of adherence to surveillance protocols regardless of the risk category. However, it is particularly imperative amongst the high-risk group, as our data shows that the PR rate in the compliant group is zero while it is $100 \%(\mathrm{P}<0.001)$ in the non-compliant intermediate group and $56.4 \%(\mathrm{P}=$ 0.001 ) in the non-compliant high-risk group.

The National Institute for Health and Care Excellence guidelines (NICE guidelines) recommend a two-week wait pathway to diagnose bladder cancer, especially if haematuria is the main presentation. ${ }^{15}$ Our results showed that half of the patients had a delay in diagnosis despite having haematuria as the most prevalent presentation in our cohort, which may also contribute to the overall bad prognosis. Furthermore, at the time of the first resection, $90 \%$ failed to have IV-MMC which may explain the high RR in all subgroup, as immediate postoperative IV-MMC significantly reduces the RR compared to TURBT alone. ${ }^{16}$ It is obvious that missing surveillance stages during NMIBC management is associated with the poorer outcome, as our scoring model shows that scoring three or more is significantly associated with higher recurrence, progression, and mortality. The KM curve shows that compliant patients had better survival than the non-compliant group over the 5 year follow-up period.

The high-risk group carries the highest risk of progression. Therefore, this category has been thoroughly evaluated to decrease the risk of progression and improve overall prognosis. Several strategies have been recommended to treat this group, such as full-dose intravesical BCG for one to three years, creation of a subgroup of highest risk tumours by EAU, and upfront cystectomy in some cases. ${ }^{4,17,18}$ In our study, the group 2 non-compliant high-risk group is associated with a significant PR and a 25\% MR. Furthermore, non-compliance to both 
cystoscopy follow-up and intravesical BCG is associated with significantly higher PR and MR. Thus, adherence to a surveillance protocol and intravesical treatment in the high-risk group is strongly recommended and should be followed strictly. More importantly, this will help to answer the trending question in the literature regarding the importance of surveillance cystoscopies during the COVID-19 pandemic. $^{19}$

\section{Strengths and Limitations of the Study}

Although this is the first and most well-characterized NMIBC cohort in the Arab world, one of the limitations we face here is the limited sample size in the subgroup studies. The small number of participants limits the accuracy of the prediction model, including the propensity score matching and the statistical power of the scoring model in predicting adverse outcomes. Given these promising findings, validation in a prospective multicenter setting is warranted to evaluate the practicality and reliability of this model in predicting recurrence, progression, and mortality in high-risk patients with weak enforcement. In addition, this study has other limitations such as a few patients being lost to follow-up, and a lack of standardization in pathology reporting due to the retrospective nature of the study.

\section{Conclusions}

NMIBC is a potentially controllable disease with an excellent outcome if a surveillance protocol is applied and followed. However, this study shows that noncompliance with such protocols leads to significantly worse outcomes. RR, PR, and MR are all higher in the non-compliance group.

\section{Acknowledgment}

Many thanks to the Clinical Research Centre of An-Najah National University Hospital for the constant support and wise advice. The authors thank Samantha Kearley from the Bristol Urological Institute, UK for English editing of the manuscript.

\section{Author Contributions}

All authors made substantial contributions to conception and design, acquisition of data, or analysis and interpretation of data; took part in drafting the article or revising it critically for important intellectual content; agreed to submit to the current journal; gave final approval of the version to be published; and agree to be accountable for all aspects of the work.

\section{Funding}

No specific grant was received from any public, commercial, or non-profit funding agency for this research.

\section{Disclosure}

The authors report no conflicts of interest for this work.

\section{References}

1. Ferlay J, Soerjomataram I, Dikshit R, et al. Cancer incidence and mortality worldwide: sources, methods and major patterns in GLOBOCAN 2012. Int $J$ Cancer. 2015;136(5):E359-386. doi:10.10 02/ijc. 29210

2. Larsson SC, Andersson SO, Johansson JE, Wolk A. Diabetes mellitus, body size and bladder cancer risk in a prospective study of Swedish men. Eur J Cancer. 2008;44(17):2655-2660. doi:10.1016/ j.ejca.2008.07.012

3. Comperat E, Larre S, Roupret M, et al. Clinicopathological characteristics of urothelial bladder cancer in patients less than 40 years old. Virchows Arch. 2015;466(5):589-594. doi:10.1007/s00428-0151739-2

4. Babjuk M, Bohle A, Burger M, et al. EAU guidelines on non-muscleinvasive urothelial carcinoma of the bladder: update 2016. Eur Urol. 2017;71(3):447-461. doi:10.1016/j.eururo.2016.05.041

5. Sylvester RJ, van der Meijden AP, Oosterlinck W, et al. Predicting recurrence and progression in individual patients with stage Ta T1 bladder cancer using EORTC risk tables: a combined analysis of 2596 patients from seven EORTC trials. Eur Urol. 2006;49(3):466. doi:10.1016/j.eururo.2005.12.031

6. Liedberg F, Anderson H, Mansson A, Mansson W. Diagnostic delay and prognosis in invasive bladder cancer. Scand J Urol Nephrol. 2003;37(5):396-400. doi:10.1080/00365590310006246

7. Wallace DM, Bryan RT, Dunn JA, Begum G, Bathers S; West Midlands Urological Research Group. Delay and survival in bladder cancer. $B J U$ Int. 2002;89(9):868-878. doi:10.1046/j.1464-410X.2002.02776.x

8. Mielczarek L, Zapala P, Krajewski W, et al. Diagnostic and treatment delays among patients with primary bladder cancer in Poland: a survey study. Cent Eur J Urol. 2020;73(2):152-159. doi:10.5173/ ceju. 2020.0158

9. Abushamma F, Jaradat A, Al-azab R, et al. Recommendations and algorithm to deal with urological surgeries in the Middle East during COVID-19 Pandemic: review article and regional expert opinion. Pal Med Pharm J. 2020;5(2):133-139.

10. Huang C, Wang Y, Li X, et al. Clinical features of patients infected with 2019 novel coronavirus in Wuhan, China. Lancet. 2020;395 (10223):497-506. doi:10.1016/S0140-6736(20)30183-5

11. Taylor J, Becher E, Steinberg GD. Update on the guideline of guidelines: non-muscle-invasive bladder cancer. BJU Int. 2020;125 (2):197-205. doi:10.1111/bju.14915

12. Ritch CR, Velasquez MC, Kwon D, et al. Use and Validation of the AUA/SUO risk grouping for nonmuscle invasive bladder cancer in a contemporary Cohort. J Urol. 2020;203(3):505-511. doi:10.1097/ JU.0000000000000593

13. Witjes JA. Follow-up in non-muscle invasive bladder cancer: facts and future. World J Urol. 2020. doi:10.1007/s00345-020-03569-2

14. Datovo JCF, Neto WA, Mendonca GB, Andrade DL, Reis LO. Prognostic impact of non-adherence to follow-up cystoscopy in non-muscle-invasive bladder cancer (NMIBC). World $J$ Urol. 2019;37(10):2067-2071. doi:10.1007/s00345-019-02697-8 
15. National Institute for Health and Care Excellence (NICE). Suspected cancer: recognition and referral; NICE guideline [NG12]; 2015. Available from: https://www.nice.org.uk/guidance/ng12/chapter/ 1-Recommendations-organised-by-site-of-cancer\#urological-cancers. Accessed June 13, 2020.

16. Sylvester RJ, Oosterlinck W, Holmang S, et al. Systematic review and individual patient data meta-analysis of randomized trials comparing a single immediate instillation of chemotherapy after transurethral resection with transurethral resection alone in patients with stage pta-pt1 urothelial carcinoma of the bladder: which patients benefit from the instillation? Eur Urol. 2016;69(2):231-244. doi:10.1016/j.eururo.2015.05.050
17. Sylvester RJ, van der MA, Lamm DL. Intravesical bacillus Calmette-Guerin reduces the risk of progression in patients with superficial bladder cancer: a meta-analysis of the published results of randomized clinical trials. J Urol. 2002;168(5):1964-1970. doi:10.1016/S0022-5347(05)64273-5

18. Faba OR, Palou J, Breda A, Villavicencio H. High-risk non-muscleinvasive bladder cancer: update for a better identification and treatment. World J Urol. 2012;30(6):833-840. doi:10.1007/s00345-012-0967-1

19. Mostafid H. Cystoscopic surveillance for bladder cancer: learning the lessons forced upon us by the Covid-19 pandemic. Scand J Urol. 2020;1-2. doi:10.1080/21681805.2020.1794956

\section{Publish your work in this journal}

Cancer Management and Research is an international, peer-reviewed open access journal focusing on cancer research and the optimal use of preventative and integrated treatment interventions to achieve improved outcomes, enhanced survival and quality of life for the cancer patient.
The manuscript management system is completely online and includes a very quick and fair peer-review system, which is all easy to use. Visit http://www.dovepress.com/testimonials.php to read real quotes from published authors. 